\title{
37.
}

\section{ON THE ROTATION OF A SOLID BODY ROUND A FIXED POINT.}

[From the Cambridge and Dublin Mathematical Journal, vol. I. (1846), pp. 167-173 and 264-274.]

ThE difficulty of completing elegantly the solution of this problem, in the case where no forces act upon the body, arises from the complexity and want of symmetry of the ordinary formulæ for determining the position of one set of rectangular axes with respect to another set; in consequence of which it has hitherto been considered necessary to make a particular supposition relative to the position of the fixed axes in space, viz. that one of them shall be perpendicular to the "invariable plane" of the rotating body. But some formulæ for the above purpose, given also by Euler, are entirely free from these objections. Imagine two sets of axes $A x, A y, A z, A x_{1}, A y_{1}, A z_{\text {r }}$. The former set can be made to coincide with the second set, by a rotation $\theta$ round a certain axis $A R$, inclined to $A x, A y, A z$ at angles $f, g, h$. (As usual $f, g, h$ are the angles $R A x, R A y, R A z$ considered as positive, and the rotation is in the same direction as a rotation round $A z$ from $x$ towards $y$.) This axis may be termed the resultant axis, and the angle $\theta$ the resultant rotation. The formulæ of Euler express the coefficients of the transformation in terms of the resultant rotation and of the position of the resultant axis, i.e. in terms of $\theta$ and of the angles $f, g, h$, whose cosines are connected by the equation

$$
\cos ^{2} f+\cos ^{2} g+\cos ^{2} h=1 .
$$

'This idea was improved upon by M. Rodrigues (Liouv. tom. v. p. 404), who introduced the quantities

$$
\tan \frac{1}{2} \theta \cos f, \quad \tan \frac{1}{2} \theta \cos g, \quad \tan \frac{1}{2} \theta \cos h,
$$

(quantities which will be represented by $\lambda, \mu, \nu$ ) by means of which he expressed the 
coefficients as fractions, the numerators of which are very simple rational functions of the second order of $\lambda, \mu, \nu$, and which have the common denominator $\left(1+\lambda^{2}+\mu^{2}+\nu^{2}\right)$. These quantities may conveniently be termed the "coordinates of the resultant rotation," and the denominator or the square of the secant of the semi-angle of resultant rotation will be the "modulus" of the rotation. The elegance of these results led me to apply them to the mechanical question, and I gave in the Journal (vol. III. p. 224), [6], the differential equations of motion obtained in terms of $\lambda, \mu, \nu$ : which I integrated as in the common theory, by supposing one of the fixed axes to be perpendicular to the invariable plane. Though my attention was again called to the subject, by the connexion of some of these formulæ with Sir William Hamilton's theory of quaternions, no other way of performing the integration occurred to me. The grand discovery however of Jacobi, of the possibility of reducing to quadratures the two final differential equations of any mechanical problem, when the remaining integrals are known, induced me to resume the problem, and at least attempt to bring it so far as to obtain a differential equation of the first order between two variables only, the multiplier of which could be obtained theoretically by Jacobi's discovery. The choice of two new variables to which the equations of the problem led me, enabled me to effect this with the greatest simplicity; and the differential equation which I finally obtained, turned out to be integrable per se, so that the laborious process of finding the multiplier became unnecessary. The new variables $\Omega, v$ have the following geometrical interpretations, $\Omega=k \tan \frac{1}{2} \theta \cos I$, where $k$ is the principal moment, $\theta$ as before the angle of resultant rotation, and $I$ is the inclination of the resultant axis to the perpendicular upon the invariable plane, and $v=k^{2} \cos ^{2} \frac{1}{2} J$; where, if we imagine a line $A Q$ having the same position relatively to the axes in fixed space that the perpendicular upon the invariable plane has to the principal axes of the rotating body, then $J$ is the inclination of this line to the above perpendicular. To the choice of these variables I was led by the analysis only. It will be seen that $p, q, r$ are functions of $v$ only, while $\lambda, \mu, \nu$ contain besides the variable $\Omega$. In obtaining these relations a singular equation $\Omega^{2}=\kappa v-k^{2}$ occurs (equation 13), which may also be written $1+\tan ^{2} \frac{1}{2} \theta \cos ^{2} I=\sec ^{2} \frac{1}{2} \theta \cos ^{2} \frac{1}{2} J$, in which form the interpretation of the quantities $I, J$ has just been given. The equation (17), it may be remarked, is self-evident: it expresses that the inclination of the resultant axis to the normal of the invariable plane, is equal to the inclination of the same axis to the line $A Q$. Now the resultant axis having the same inclination to the axes fixed in space as it has to the principal axes, and the line $A Q$ the same inclinations to these fixed axes that the normal to the invariable plane has to the principal axes, the truth of the proposition becomes manifest. The correspondence in form between the systems (10) and (14) is also worth remarking. The final results at which $I$ arrive are, that the time and the are whose tangent is $\Omega \div k$, are each of them expressible as the integrals of certain algebraical functions of $v$. The notation throughout is the same as that made use of in the paper already quoted.

The equations of rotatory motion are

$$
d t=\frac{d p}{P}=\frac{d q}{Q}=\frac{d r}{R}=\frac{d \lambda}{\Lambda}=\frac{d \mu}{\mathrm{M}}=\frac{d \nu}{\mathrm{N}}
$$


where

$$
\begin{gathered}
P=\frac{1}{A}\left[(B-C) q r+\frac{1}{2}\left\{\left(1+\lambda^{2}\right) \frac{d V}{d \lambda}+(\lambda \mu+\nu) \frac{d V}{d \mu}+(\lambda \nu-\mu) \frac{d V}{d \nu}\right\}\right], \\
\left.Q=\frac{1}{B}\left[(C-A) r p+\frac{1}{2}\left\{(\mu \lambda-\nu) \frac{d V}{d \lambda}+\left(1+\mu^{2}\right) \frac{d V}{d \mu}+(\mu \nu+\lambda) \frac{d V}{d \nu}\right\}\right],\right\} \\
R=\frac{1}{C}\left[(A-B) p q+\frac{1}{2}\left\{(\nu \lambda+\mu) \frac{d V}{d \lambda}+(\mu \nu-\lambda) \frac{d V}{d \mu}+\left(1+\nu^{2}\right) \frac{d V}{d \nu}\right\}\right], \\
\Lambda=\frac{1}{2}\left\{\left(1+\lambda^{2}\right) p+(\lambda \mu-\nu) q+(\lambda \nu+\mu) r\right\}, \\
M=\frac{1}{2}\left\{(\mu \lambda+\nu) p+\left(1+\mu^{2}\right) q+(\mu \nu-\lambda) r\right\}, \\
\mathrm{N}=\frac{1}{2}\left\{(\nu \lambda-\mu) p+(\mu \nu+\lambda) q+\left(1+\nu^{2}\right) r\right\},
\end{gathered}
$$

[whence also

$$
\lambda \Lambda+\mu \mathrm{M}+\nu \mathrm{N}=\frac{1}{2} \kappa(\lambda p+\mu q+\nu r) .
$$

In the case where the forces vanish, the first three equations become simply

$$
\begin{aligned}
P & =\frac{1}{A}(B-C) q r \\
Q & =\frac{1}{B}(C-A) r p \\
R & =\frac{1}{C}(A-B) p q,
\end{aligned}
$$

and here the usual four integrals of the system are

$$
A p^{2}+B q^{2}+C r^{2}=h
$$

$$
\left.\begin{array}{l}
A p\left(1+\lambda^{2}-\mu^{2}-\nu^{2}\right)+2 B q(\lambda \mu-\nu)+2 C r(\nu \lambda+\mu)=\mathrm{a}\left(1+\lambda^{2}+\mu^{2}+\nu^{2}\right), \\
2 A p(\lambda \mu+\nu)+B q\left(1+\mu^{2}-\nu^{2}-\lambda^{2}\right)+2 C r(\mu \nu-\lambda)=\mathrm{b}\left(1+\lambda^{2}+\mu^{2}+\nu^{2}\right) \\
2 A p(\nu \lambda-\mu)+2 B q\left(\mu \nu^{\prime}+\lambda\right)+C r\left(1+\nu^{2}-\lambda^{2}-\mu^{2}\right)=\mathrm{c}\left(1+\lambda^{2}+\mu^{2}+\nu^{2}\right),
\end{array}\right\}
$$

or as they may also be written,

$$
\left.\begin{array}{l}
\mathrm{a}\left(1+\lambda^{2}-\mu^{2}-\nu^{2}\right)+2 \mathrm{~b}(\lambda \mu+\nu)+2 \mathrm{c}(\nu \lambda-\mu)=A p\left(1+\lambda^{2}+\mu^{2}+\nu^{2}\right), \\
2 \mathrm{a}(\lambda \mu-\nu)+\mathrm{b}\left(1+\mu^{2}-\nu^{2}-\lambda^{2}\right)+2 \mathrm{c}(\mu \nu+\lambda)=B q\left(1+\lambda^{2}+\mu^{2}+\nu^{2}\right), \\
2 \mathrm{a}(\nu \lambda+\mu)+2 \mathrm{~b}(\mu \nu-\lambda)+\mathrm{c}\left(1+\nu^{2}-\lambda^{2}-\mu^{2}\right)=C r\left(1+\lambda^{2}+\mu^{2}+\nu^{2}\right),
\end{array}\right\}
$$

to which we may add,

$$
A^{2} p^{2}+B^{2} q^{2}+C^{2} r^{2}=k^{2}
$$

where

$$
k^{2}=a^{2}+b^{2}+c^{2}
$$

Introducing the quantities $\kappa, \Omega$, (the former of which has been already made use of) given by the equations

$$
\left.\begin{array}{l}
\kappa=1+\lambda^{2}+\mu^{2}+\nu^{2}, \\
\Omega=\lambda A p+\mu B q+\nu C r
\end{array}\right\}
$$


The equations (6) may be written under the form

$$
\left.\begin{array}{r}
2 \lambda \Omega+2 \mu C r-2 \nu B q=\kappa(A p+a)-2 A p, \\
-2 \lambda C r+2 \mu \Omega+2 \nu A p=\kappa(B q+b)-2 B q, \\
2 \lambda B q-2 \mu A p+2 \nu \Omega=\kappa(C r+c)-2 C r
\end{array}\right\}
$$

whence also, multiplying by $A p, B q, C r$, and adding,

$$
2 \Omega^{2}=\kappa\left\{k^{2}+(A p \mathrm{a}+B q \mathrm{~b}+C r \mathrm{c})\right\}-2 k^{2}
$$

or writing

$$
k^{2}+(A p \mathrm{a}+B q \mathrm{~b}+C r \mathrm{c})=2 v
$$

this becomes

$$
\Omega^{2}=\kappa v-k^{2}
$$

an equation, the geometrical interpretation of which has already been given.

From the equations (10) we deduce the inverse system

$$
\left.\begin{array}{r}
a \Omega-b C r+c B q=2 \lambda v-\Omega A p \\
a C r+b \Omega-c A p=2 \mu v-\Omega B q \\
-a B q+b A p+c \Omega=2 \nu v-\Omega C r
\end{array}\right\}
$$

which are easily verified by multiplying by $\Omega, C r,-B q$; or by $-C r, \Omega, A p$; or $B q,-A p, \Omega$ : adding and reducing, by which means the equations (10) are re-obtained. Hence also if for shortness

$$
\left.\begin{array}{l}
\Phi=\mathrm{a} p+\mathrm{b} q+\mathrm{cr}, \\
\nabla=\mathrm{a} q r(B-C)+\mathrm{b} r p(C-A)+\mathrm{c} p q(A-B),
\end{array}\right\}
$$

we have, multiplying by $p, q, r$, and adding,

$$
\Omega \Phi-\nabla=2 v(\lambda p+\mu q+\nu r)-\Omega h .
$$

To these may be added the equation

$$
\Omega=a \lambda+b \mu+c \nu
$$

which follows immediately from either of the systems (10) or (14).

We may also put the equations (10) under this other form,

$$
\left.\begin{array}{rl}
2 \lambda \Omega-2 \mu \mathrm{c}+2 \nu \mathrm{b} & =\kappa(A p+\mathrm{a})-2 \mathrm{a}, \\
2 \lambda \mathrm{c}+2 \mu \Omega-2 \nu \mathrm{a} & =\kappa(B q+\mathrm{b})-2 \mathrm{~b}, \\
-2 \lambda \mathrm{b}+2 \mu \mathrm{a}+2 \nu \Omega & =\kappa(C r+\mathrm{c})-2 \mathrm{c},
\end{array}\right\}
$$

It may be remarked now, that $p, q, r$ are functions of $v$; since we have to determine these quantities, the three equations

$$
\left.\begin{array}{l}
A p^{2}+B q^{2}+C r^{2}=h, \\
A^{2} p^{2}+B^{2} q^{2}+C^{2} r^{2}=k^{2}, \\
A p \mathrm{a}+B q \mathrm{~b}+C r \mathrm{c}=2 v-k^{2},
\end{array}\right\}
$$


Also $\lambda, \mu, \nu$ are given by the equations (14) as functions of $p, q, r, \Omega$, i.e. of $v, \Omega$; so that every thing is prepared for the investigation of the differential equation between $v, \Omega$. To find this we have immediately

$$
d v=\frac{1}{2}(A \mathrm{a} d p+B \mathrm{~b} d q+C \mathrm{c} d r)=\frac{1}{2} \nabla d t
$$

from the equations (4) and (15). $\nabla$ is of course to be considered as a given function of $v$. Again,

where

$$
\begin{gathered}
\Omega d \Omega=\frac{1}{2}(\kappa d v+v d \kappa) \\
d \kappa=2(\lambda d \lambda+\mu d \mu+\nu d \nu)
\end{gathered}
$$

or from the equations (1), (3), [and (3 bis)],

$$
d \kappa=\kappa(\lambda p+\mu q+\nu r) d t .
$$

Hence, from (16),

$$
\begin{gathered}
2 v d \kappa=\kappa \\
2(\Omega d \kappa+\kappa d v)=\kappa \Omega(h+\Phi) d t \ldots \\
d \Omega=\frac{1}{4} \kappa(h+\Phi) d t, \\
=\frac{1}{4} \frac{\Omega^{2}+k^{2}}{v}(h+\Phi) d t
\end{gathered}
$$

and therefore, from (19),

$$
\frac{2 d \Omega}{\Omega^{2}+k^{2}}=\frac{h+\Phi}{v \nabla} d v
$$

the required differential equation, in which $\Phi, \nabla$ are given functions of $v$, i.e. they are functions of $p, q, r$ by the equations (15), and these quantities are functions of $v$ by (18). The variables in (26) are therefore separated, and we have the integral equation

$$
2 \tan ^{-1} \frac{\Omega}{k}=\delta+k \int \frac{(h+\Phi) d v}{v \nabla}
$$

where $\delta$ is the constant of integration. The equation (19) gives also

$$
t-\epsilon=2 \int \frac{d v}{\nabla}
$$

and thus the solution of the problem is completely effected. The integrals may be taken from any particular value $v_{0}$ of $v$. The variable $\Omega$ may be exhibited as the integral of an explicit algebraical function, by recurring to the variable $\phi$ of the paper quoted.

Thus if

$$
\begin{aligned}
& A p_{0}{ }^{2}+B q_{0}{ }^{2}+C r_{0}{ }^{2}=h, \\
& A^{2} p_{0}{ }^{2}+B^{2} q_{0}{ }^{2}+C^{2} r_{0}{ }^{2}=k^{2}, \\
& A p_{0} \mathrm{a}+B q_{0} \mathrm{~b}+C r_{0} \mathrm{c}=2 v_{0}-k^{2}
\end{aligned}
$$

C. 
then the values of $p, q, r$ are respectively

$$
\sqrt{ }\left\{p_{0}{ }^{2}-\frac{1}{A}(C-B) \phi\right\}, \quad \sqrt{ }\left\{q_{0}{ }^{2}-\frac{1}{A}(A-C) \phi\right\}, \quad \sqrt{ }\left\{r_{0}{ }^{2}-\frac{1}{C^{x}}(B-A) \phi\right\},
$$

where

$$
d t=\frac{1}{2} \frac{d \phi}{p q r}=\frac{2 d v}{\nabla}, \quad \text { or } \frac{d v}{\nabla}=\frac{1}{4} \frac{d \phi}{p q r}
$$

and then

$$
\text { 4. } \tan ^{-1} \frac{\Omega}{k}=2 \delta+k \int_{0} \frac{(h+\mathrm{a} p+\mathrm{b} q+\mathrm{cr}) d \phi}{\left(k^{2}+A p \mathrm{a}+B q \mathrm{~b}+C r \mathrm{c}\right) p q r} \text {, }
$$

in which form it is exactly analogous to the equation there obtained, p. $230,[6$, p. 34$]$

$$
4 \tan ^{-1} \nu_{0}=\int \frac{(h+k r) d \phi}{(k+C r) p q r} .
$$

On the Variation of the Constants, when the body is acted upon by Forces.

The dynamical equations of a problem being expressed in the form

$$
\begin{aligned}
& \frac{d}{d t} \frac{d T}{d \lambda^{\prime}}-\frac{d T}{d \lambda}=\frac{d V}{d \lambda}, \\
& \frac{d}{d t} \frac{d T}{d \mu^{\prime}}-\frac{d T}{d \mu}=\frac{d V}{d \mu}, \\
& \frac{d}{d t} \frac{d T}{d \nu^{\prime}}-\frac{d T}{d \nu}=\frac{d V}{d \nu},
\end{aligned}
$$

suppose the equations obtained from these by neglecting the function $V$, are integrated; each of the six integrals may be expressed in the form

$$
a=f\left(\lambda, \mu, \nu, \lambda^{\prime}, \mu^{\prime}, \nu^{\prime}, t\right),
$$

where $a$ denotes any one of the arbitrary constants. Assume

$$
\frac{d T}{d \lambda^{\prime}}=u, \quad \frac{d T}{d \mu^{\prime}}=v, \quad \frac{d T}{d \nu}=w ;
$$

then $\lambda^{\prime}, \mu^{\prime}, \nu^{\prime}$ may be expressed in terms of $\lambda, \mu, \nu, u, v, w$, and the integrals may be reduced to the form

$$
a=F(\lambda, \mu, \nu, u, v, w, t) .
$$

These equations may be considered as the integrals of the proposed system, taking into account the terms involving $V$, provided the constants [say $a, b, c, d, e, f$ ] be sup- 
posed to become variable. We have, in this case, by Lagrange's theory of the variation of the arbitrary constants, the formulæ

$$
\frac{d a}{d t}=(a, b) \frac{d V}{d b}+(a, c) \frac{d V}{d c}+(a, d) \frac{d V}{d d}+(a, e) \frac{d V}{d e}+(a, f) \frac{d V}{d f}
$$

where

$$
(a, b)=\left(\frac{d a}{d u} \frac{d b}{d \lambda}-\frac{d a}{d \lambda} \frac{d b}{d u}\right)+\left(\frac{d a}{d v} \frac{d b}{d \mu}-\frac{d a}{d \mu} \frac{d b}{d v}\right)+\left(\frac{d a}{d w} \frac{d b}{d \nu}-\frac{d a}{d \nu} \frac{d b}{d w}\right),
$$

and in which $V$ is supposed to be expressed as a function of $a, b, c, d, e, f, t$.

Thus the solution of the problem requires the calculation of thirty coefficients $(a, b)$, or rather of fifteen only, since evidently $(a, b)=-(b, a)$. It is known that these coefficients are functions of $a, b, c, d, e, f$, without $t$; so that, in calculating them, any assumed arbitrary value, e.g. $t=0$, may be given to the time.

In practice, it often happens that one of the arbitrary constants, e.g. $a$, may be expressed in the form

$$
a=F(\lambda, \mu, \nu, u, v, w, t, b, c, d, e, f),
$$

where $b, c, d, e, f$ are given functions of $\lambda, \mu, \nu, u, v, w, t$. In this case, it is easily seen that we may write

$$
(a, b)=\{(a, b)\}+(c, b) \frac{d a}{d c}+(d, b) \frac{d a}{d d}+(e, b) \frac{d a}{d e}+(f, b) \frac{d a}{d f},
$$

where, in the calculation of $\{(a, b)\}$, the differentiations upon $a$ are performed without taking into account the variability of $b, c \ldots \ldots$

In the particular problem in question, the following are the values of the new variables $u, v, w$ (Math. Journal, memoir already quoted, [6]),

$$
\begin{aligned}
& u=\frac{2}{\kappa}(\quad A p-\nu B q+\mu C r), \\
& v=\frac{2}{\kappa}(\nu A p+B q-\lambda C r), \\
& w=\frac{2}{\kappa}(-\mu A p+\lambda B q+C r) ;
\end{aligned}
$$

equations which may also be expressed in the form

$$
\begin{aligned}
& 2 A p=\left(1+\lambda^{2}\right) u+(\lambda \mu+\nu) v+(\nu \lambda-\mu) w \\
& 2 B q=(\lambda \mu-\nu) u+\left(1+\mu^{2}\right) v+(\mu \nu+\lambda) w, \\
& 2 C r=(\nu \lambda+\mu) u+(\mu \nu-\lambda) v+\left(1+\nu^{2}\right) w,
\end{aligned}
$$

or putting for shortness

$$
\lambda u+\mu v+\nu w=\varpi
$$


these become

$$
\begin{aligned}
& 2 A p=\lambda \varpi+u+\nu v-\mu w, \\
& 2 B q=\mu \varpi-\nu u+v+\lambda w, \\
& 2 C r=\nu \varpi+\mu u-\lambda v+w,
\end{aligned}
$$

whence also

$$
2 \Omega=\kappa \varpi
$$

Substituting the values of $A p, B q, C r$, given by (30) in the equations (6), we deduce

$$
\begin{aligned}
& 2 \mathrm{a}=\lambda \varpi+u-\nu v+\mu w . \\
& 2 \mathrm{~b}=\mu \varpi+\nu u+v-\lambda w, \\
& 2 \mathrm{c}=\nu \varpi-\mu u+\lambda v+w,
\end{aligned}
$$

whence also

$$
2(a \lambda+b \mu+c \nu)=\kappa \varpi
$$

which in fact follows from (33) and (17). And likewise the inverse system,

$$
\begin{aligned}
& u=\frac{2}{\kappa}(\mathrm{a}+\nu \mathrm{b}-\mu \mathrm{c}) \\
& v=\frac{2}{\kappa}(-\nu \mathrm{a}+\mathrm{b}+\lambda \mathrm{c}), \\
& w=\frac{2}{\kappa}(\mu \mathrm{a}-\lambda \mathrm{b}+\mathrm{c}) .
\end{aligned}
$$

It is easy to deduce

$$
\begin{aligned}
k^{2} & =\frac{1}{4} \kappa\left[u^{2}+v^{2}+w^{2}+\varpi^{2}\right] \\
v & =\frac{1}{4}\left[\left(u^{2}+v^{2}+w^{2}\right)+(1+\kappa) \varpi^{2}\right]
\end{aligned}
$$

Again, from the equations (10 bis),

$$
\begin{aligned}
\kappa(\mathrm{b} C r-\mathrm{c} B q) & =-2 \lambda\left(\mathrm{a}^{2}+\mathrm{b}^{2}+\mathrm{c}^{2}\right)+2 \mathrm{a}(\lambda \mathrm{a}+\mu \mathrm{b}+\nu \mathrm{c})+2(\mathrm{~b} \nu-\mathrm{c} \mu) \Omega \\
& =-2 \lambda k^{2}+2(\mathrm{a}+\mathrm{b} \nu-\mathrm{c} \mu) \Omega \\
& =-2 \lambda k^{2}+\kappa u \Omega
\end{aligned}
$$

and, forming also the similar expressions for $\kappa(\mathrm{c} A p-\mathrm{a} C r)$, and $\kappa(\mathrm{a} B q-\mathrm{b} A p)$, we thus obtain

$$
\begin{aligned}
& \Omega u-\frac{2}{\kappa} k^{2} \lambda=\mathrm{b} C r-\mathrm{c} B q \\
& \Omega v-\frac{2}{\kappa} k^{2} \mu=\mathrm{c} A p-\mathrm{b} C r \\
& \Omega w-\frac{2}{\kappa} k^{2} \nu=\mathrm{a} B q-\mathrm{c} A p
\end{aligned}
$$

to which many others might probably be joined.

The constants of the problem are $\mathrm{a}, \mathrm{b}, \mathrm{c}, h, \epsilon, \delta$. Of these $\mathrm{a}, \mathrm{b}, \mathrm{c}$ are given as functions of $\lambda, \mu, \nu, u, v, w$, by the equations (34); in which $\sigma$ is to be considered as 
standing for $\lambda u+\mu v+\nu w$. \{These determine $k^{2}$, which is however given immediately by (37).\} As for $h$, we have

$$
h=\frac{1}{A}(A p)^{2}+\frac{1}{B}(B q)^{2}+\frac{1}{C}(C r)^{2},
$$

where $A p, B q, C r$ are given as functions of $\lambda, \mu, \nu, u, v, w$ by (32), in which also $\varpi$ stands for $\lambda u+\mu v+\nu w$. Again,

$$
\begin{gathered}
\epsilon=t-\frac{1}{2} \int \frac{d v}{\nabla} \\
\delta=2 \tan ^{-1} \frac{\kappa \varpi}{2 k}-\frac{1}{4} k \int \frac{(h+\Phi) d v}{v \nabla}
\end{gathered}
$$

in each of which $\nabla, \Phi$ are functions of $v$, and of a, b, c, h, partly as entering explicitly into these functions, partly as contained implicitly in $p, q, r$, which enter into $\nabla, \Phi$, and are functions of $v, h, k$ given by (18). After the integration $v$ is to be considered.a function of $\lambda, \mu, \nu, u, v, w$ given by (38). Both of the integrals may be supposed taken from a certain value $v_{0}$ of $v$, which may be considered as an absolutely invariable arbitrary constant, since without it we have the right number, six, of arbitrary constants.

First to find $(a, b),(b, c)$, and (c, a). From (34) we have

$$
\begin{aligned}
&(\mathrm{a}, \mathrm{b})=\frac{1}{4}\{\left(1+\lambda^{2}\right)(\mu u-w)-(\lambda u+\varpi)(\lambda \mu+\nu) \\
&+(\lambda \mu-\nu)(\mu v+\varpi)-(\lambda v+w)\left(1+\mu^{2}\right) \\
&+(\nu \lambda+\mu)(u+\mu w)-(\lambda w-v)(\mu \nu-\lambda)\} \\
&=\frac{1}{2}(\mu u-\lambda v-w-\nu \varpi)=-\frac{1}{2} 2 \mathrm{c}=-\mathrm{c}
\end{aligned}
$$

whence the system

$$
(b, c)=-a, \quad(c, a)=-b, \quad(a, b)=-c
$$

Also we may add $\quad(k, a)=\frac{a}{k}(a, a)+\frac{b}{k}(b, a)+\frac{c}{k}(c, a)=0$,

or

$$
(k, \mathrm{a})=0, \quad(k, \mathrm{~b})=0, \quad(k, \mathrm{c})=0
$$

which will be useful in calculating some of the following coefficients.

Proceeding to calculate $(\mathrm{a}, h),(\mathrm{b}, h),(\mathrm{c}, h)$. It is seen immediately that

$$
(\mathrm{a}, h)=2\{p(\mathrm{a}, A p)+q(\mathrm{a}, B q)+r(\mathrm{a}, C r)\},
$$

where $A p, B q, C r$, are given by the equations (32), so that

$$
\begin{aligned}
(\mathrm{a}, A p)=\frac{1}{4}\{ & \left(1+\lambda^{2}\right)(\lambda u+\varpi)-\left(1+\lambda^{2}\right)(\lambda u+\varpi) \\
+ & (\lambda \mu-\nu)(\lambda v-w)-(\lambda \mu+\nu)(\lambda v+w) \\
+ & (\nu \lambda+\mu)(v+\lambda w)-(\nu \lambda-\mu)(-v+\lambda w)\}
\end{aligned}
$$

i.e.

$($ a, $A p)=0$. 


$$
\text { Similarly } \quad \begin{aligned}
(\mathrm{a}, B q)=\frac{1}{4}\{ & \left(1+\lambda^{2}\right)(\mu u+w)-(\lambda u+\varpi)(\lambda \mu-\nu) \\
+ & (\lambda \mu-\nu)(\mu v+\varpi)-(\lambda v+w)\left(1+\mu^{2}\right) \\
+ & (\nu \lambda+\mu)(\mu w-u)-(-v+\lambda w)(\mu \nu+\lambda)\}
\end{aligned}
$$

i.e. $\quad(a, B q)=0$,

and similarly $\quad(\mathrm{a}, \mathrm{Cr})=0$;

whence

$(\mathrm{a}, h)=0$, and therefore $(\mathrm{b}, h)=0,(\mathrm{c}, h)=0$

also

$(k, h)=0$,

Next we have to determine $(a, \epsilon),(b, \epsilon),(c, \epsilon)$. Here $\epsilon$ being a function of $u, v, w, \lambda, \mu, \nu, \mathrm{a}, \mathrm{b}, \mathrm{c}, h$, we must write

i.e.

$$
(\mathrm{a}, \epsilon)=\{(\mathrm{a}, \epsilon)\}+(\mathrm{a}, \mathrm{b}) \frac{d \epsilon}{d \mathrm{~b}}+(\mathrm{a}, \mathrm{c}) \frac{d \epsilon}{d \mathrm{c}}+(\mathrm{a}, h) \frac{d \epsilon}{d h}
$$$$
(a, \epsilon)=\{(a, \epsilon)\}+b \frac{d \epsilon}{d c}-c \frac{d \epsilon}{d b}
$$

But

$$
\epsilon=t-2 \int \frac{d v}{\nabla} ; \text { and thence }\{(\mathrm{a}, \epsilon)\}=-\frac{2}{\nabla}(\mathrm{a}, v)
$$

and $v$ is given immediately as a function of $\lambda, \mu, \nu, u, v, w$, by the equation (38) Hence

$$
\begin{aligned}
& (\mathrm{a}, v)=\frac{1}{4}\left[\left(1+\lambda^{2}\right)\left\{(1+\kappa) u \varpi+\lambda \varpi^{2}\right\}-(\lambda u+\varpi)\{u+\lambda(1+\kappa) \varpi\}\right. \\
& +(\lambda \mu-\nu)\left\{(1+\kappa) v \varpi+\mu \varpi^{2}\right\}-(\lambda v+w)\{v+\mu(1+\kappa) \varpi\} \\
& \left.\left.+(\nu \lambda+\mu)\left\{(1+\kappa) w \varpi+\nu \varpi^{2}\right)\right\}-(-v+\lambda w)\{w+\nu(1+\kappa) \varpi\}\right] \\
& =\frac{1}{4}\left\{(1+\kappa) \varpi u-\lambda(1+\kappa) \varpi^{2}+\lambda \kappa-\lambda\left(u^{2}+v^{2}+w^{2}\right)-u \varpi\right\} \\
& =\frac{1}{4}\left\{\kappa u \varpi-\lambda \varpi^{2}-\lambda\left(u^{2}+v^{2}+w^{2}\right)\right\} \\
& =\frac{1}{4} \kappa u \varpi-\frac{k^{2} \lambda}{\kappa}=\frac{1}{2}\left(\Omega u-\frac{2 k^{2} \lambda}{\kappa}\right)\{\text { by }(37) \text { and }(33)\} \text {, } \\
& =\frac{1}{2}(\mathrm{~b} C r-\mathrm{cBq})
\end{aligned}
$$

whence

$$
\begin{aligned}
\{(\mathrm{a}, \epsilon)\} & =-\frac{1}{\nabla}(\mathrm{b} C r-\mathrm{c} B q) \\
(\mathrm{a}, \epsilon) & =-\frac{1}{\nabla}(\mathrm{b} C r-\mathrm{c} B q)+\mathrm{b} \frac{d \epsilon}{d \mathrm{c}}-\mathrm{c} \frac{d \epsilon}{d \mathrm{~b}}
\end{aligned}
$$

The terms $\mathrm{b} \frac{d \epsilon}{d \mathrm{c}}-\mathrm{c} \frac{d \epsilon}{d \mathrm{~b}}$ are evidently of the form $F(v)-F\left(v_{0}\right)$

If therefore we suppose $v=v_{0}$, we have

$$
(\mathrm{a}, \epsilon)=-\frac{1}{\nabla_{0}}\left(\mathrm{~b} C r_{0}-\mathrm{c} B q_{0}\right)
$$


if $p_{0}, q_{0}, r_{0}, \nabla_{0}$ refer to the value $v_{0}$ of $v$, i.e. if

$$
\begin{aligned}
& A p_{0}^{2}+B q_{0}^{2}+C r_{0}^{2}=h \ldots \ldots \\
& A^{2} p_{0}^{2}+B^{2} q_{0}^{2}+C^{2} r_{0}^{2}=k^{2}, \\
& A p_{0} \mathrm{a}+B q_{0} \mathrm{~b}+C r_{0} \mathrm{c}=2 v_{0}-k^{2} .
\end{aligned}
$$

\{This implies evidently

$$
\mathrm{b} \frac{d \epsilon}{d c}-\mathrm{c} \frac{d \epsilon}{d b}=\frac{1}{\nabla}(\mathrm{b} C r-\mathrm{c} B q)-\frac{1}{\nabla_{0}}\left(\mathrm{~b} C r_{0}-\mathrm{c} B q_{0}\right),
$$

an equation which it is interesting to verify. In fact, from the value of $\epsilon$

$$
\mathrm{b} \frac{d \epsilon}{d \mathrm{c}}-\mathrm{c} \frac{d \epsilon}{d \mathrm{~b}}=-2 \int d v\left(\mathrm{~b} \frac{d}{d \mathrm{c}}-\mathrm{c} \frac{d}{d \mathrm{~b}}\right) \frac{1}{\nabla}=2 \int d v \frac{1}{\nabla^{2}}\left(\mathrm{~b} \frac{d \nabla}{d \mathrm{c}}-\mathrm{c} \frac{d \nabla}{d \mathrm{~b}}\right)
$$

or we have to show that

$$
\frac{d}{d v} \frac{1}{\nabla}(\mathrm{b} C r-\mathrm{c} B q)=\frac{2}{\nabla^{2}}\left(\mathrm{~b} \frac{d \nabla}{d \mathrm{c}}-\mathrm{c} \frac{d \nabla}{d \mathrm{~b}}\right)=\frac{2}{\nabla^{2}} \delta \nabla ;
$$

if for shortness

$$
\delta=\mathrm{b} \frac{d}{d \mathrm{c}}-\mathrm{c} \frac{d}{d \mathrm{~b}}
$$

Now $\nabla$ containing a, b, c explicitly, and also as involved in $p, q, r$, we have

$$
\delta \nabla=\mathrm{b} p q(A-B)-\operatorname{crp}(C-A)+\frac{d \nabla}{d p} \delta p+\frac{d \nabla}{d q} \delta q+\frac{d \nabla}{d r} \delta r=\mathrm{b} p q(A-B)-\operatorname{crp}(C-A)+\delta^{\prime} \nabla
$$

suppose. The equation to be verified becomes

$$
\nabla\left(\mathrm{b} C \frac{d r}{d v}-\mathrm{c} B \frac{d q}{d v}\right)-(\mathrm{b} C r-\mathrm{c} B q) \frac{d \nabla}{d v}=2\left\{\mathrm{~b} p q(A-B)-\operatorname{crp}(C-A)+\delta^{\prime} \nabla\right\} .
$$

Now, observing that $\delta k=0$, we have

$$
\begin{aligned}
& A p \delta p+B q \delta q+C r \delta r=0 \\
& A^{2} p \delta p+B^{2} q \delta q+C^{2} r \delta r=0 \\
& A \text { a } \delta p+B b \delta q+C c \delta r=-(\mathrm{b} C r-\mathrm{c} B q)
\end{aligned}
$$

Also,

$$
\begin{aligned}
& A p \frac{d p}{d v}+B q \frac{d q}{d v}+C r \frac{d r}{d v}=0 \\
& A^{2} p \frac{d p}{d v}+B^{2} q \frac{d q}{d v}+C^{2} r \frac{d r}{d v}=0 \\
& A a \frac{d p}{d v}+B b \frac{d q}{d v}+C \mathrm{c} \frac{d r}{d v}=2
\end{aligned}
$$


whence evidently

or

$$
\begin{gathered}
\frac{d p}{d v}=\frac{-2}{\mathrm{~b} C r-\mathrm{c} B q} \delta p, \quad \frac{d q}{d v}=\frac{-2}{\mathrm{~b} C r-\mathrm{c} B q} \delta q, \quad \frac{d r}{d v}=\frac{-2}{\mathrm{~b} C r-\mathrm{c} B q} \delta r \\
\frac{d \nabla}{d v}=\frac{-2}{\mathrm{~b} C r-\mathrm{c} B q} \delta^{\prime} \nabla
\end{gathered}
$$

or the equation to be verified is simply

$$
\nabla\left(\mathrm{b} C \frac{d r}{d v}-\mathrm{c} B \frac{d q}{d v}\right)=2\{\mathrm{~b} p q(A-B)-\operatorname{crp}(C-A)\}
$$

which follows immediately from the three equations just given for the determination of $\left.\frac{d p}{d v}, \frac{d q}{d v}, \frac{d r}{d v}\right\}$

From these values also

$$
(k, \epsilon)=0
$$

Next, to calculate $(h, \epsilon)$,

$$
(h, \epsilon)=\{(h, \epsilon)\}+(h, \mathrm{a}) \frac{d \epsilon}{d \mathrm{a}}+(h, \mathrm{~b}) \frac{d \epsilon}{d \mathrm{~b}}+(h, \mathrm{c}) \frac{d \epsilon}{d \mathrm{c}}
$$

but the three last terms being evidently such as to vanish for $v=v_{0}$, we may neglect them, and consider $(h, \epsilon)$ as the value which $\{(h, \epsilon)\}$ assumes for this value of $v$.

Now

$$
\{(h, \epsilon)\}=2 p\{(A p, \epsilon)\}+2 q\{(B q, \epsilon)\}+2 r\{(C r, \epsilon)\}
$$

where

$$
\{(A p, \epsilon)\}=-\frac{2}{\nabla}(A p, v)
$$

and

$$
\begin{aligned}
& (A p, v)=\frac{1}{4}\left[\left(1+\lambda^{2}\right)\left\{(1+\kappa) u \varpi+\lambda \varpi^{2}\right\}-(\lambda u+\varpi)\{u+\lambda(1+\kappa) \varpi\}\right. \\
& +(\lambda \mu+\nu)\left\{(1+\kappa) v \omega+\mu \varpi^{2}\right\}-(\lambda v-w)\{v+\mu(1+\kappa) \varpi\} \\
& \left.+(\nu \lambda-\mu)\left\{(1+\kappa) w \varpi+\nu \varpi^{2}\right\}-(v+\lambda w)\{w+\nu(1+\kappa) \varpi\}\right] \\
& =\frac{1}{4}\left\{(1+\kappa) \varpi u+\lambda \kappa \varpi^{2}-\lambda(1+\kappa) \varpi^{2}-\lambda\left(u^{2}+v^{2}+w^{2}\right)-\varpi u\right\}=(\mathrm{a}, v) \\
& =\frac{1}{2}(\mathrm{~b} C r-\mathrm{c} B q) \text {, }
\end{aligned}
$$

whence

$$
\{(A p, \epsilon)\}=-\frac{1}{\nabla}(\mathrm{b} C r-\mathrm{c} B q)
$$

and therefore

$$
\begin{aligned}
& \{(B q, \epsilon)\}=-\frac{1}{\nabla}(\mathrm{c} A p-\mathrm{a} C r) \\
& \{(C r, \epsilon)\}=-\frac{1}{\nabla}(\mathrm{a} B q-\mathrm{b} A p),
\end{aligned}
$$


whence

$$
\{(h, \epsilon)\}=-2 \text {, }
$$

and therefore

$$
(h, \epsilon)=-2
$$

Next, to find $(\mathrm{a}, \delta),(\mathrm{b}, \delta),(\mathrm{c}, \delta),(h, \delta)$,

$$
\begin{aligned}
\delta=2 & \tan ^{-1} \frac{\kappa \varpi}{2 k}-k \int \frac{(h+\Phi) d v}{v \nabla}, \\
=\delta^{\prime} & +\delta^{\prime \prime} \text { suppose, } \\
(\mathrm{a}, \delta) & =\left(\mathrm{a}, \delta^{\prime}\right)+\left(\mathrm{a}, \delta^{\prime \prime}\right), \\
\left(\mathrm{a}, \delta^{\prime}\right) & =\frac{k}{\kappa v}(\mathrm{a}, \kappa \varpi)+(\mathrm{a}, k) \frac{d \delta^{\prime}}{d k}, \\
\kappa^{2} \varpi^{2}+4 k^{2} & \left.=4\left(\Omega^{2}+k^{2}\right)=4 \kappa v\right\} \\
& =\frac{k}{\kappa v}(\mathrm{a}, \kappa \varpi),
\end{aligned}
$$

where

$$
\begin{aligned}
(\mathrm{a}, \kappa \varpi)=\frac{1}{2}\{ & \left(1+\lambda^{2}\right)(\kappa v+2 \lambda \varpi)-(\lambda u+\varpi) \kappa \lambda \\
& +(\lambda \mu-\nu)(\kappa v+2 \mu \sigma)-(\lambda v+w) \kappa \mu \\
& +(\nu \lambda+\mu)(\kappa w+2 \nu \sigma)-(-v+\lambda w) \kappa \nu\} \\
= & \frac{1}{2} \kappa(u+\lambda \varpi)=A p-\nu B q+\mu C r+\lambda \Omega=\frac{1}{2}(\mathrm{a}+A p) \kappa
\end{aligned}
$$

by equations (29), (33), and (10);

that is

$$
\left(\mathrm{a}, \delta^{\prime}\right)=\frac{k}{2 v}(\mathrm{a}+A p)
$$

Also

$$
\begin{aligned}
\left(\mathrm{a}, \delta^{\prime \prime}\right) & =-k \frac{h+\Phi}{v \nabla}(\mathrm{a}, v)+(\mathrm{a}, \mathrm{b}) \frac{d \delta^{\prime \prime}}{d \mathrm{~b}}+\& \mathrm{c} \\
& =-\frac{1}{2} k \frac{h+\Phi}{v \nabla}(\mathrm{b} C r-\mathrm{c} B q)+F v-F v_{0}
\end{aligned}
$$

whence

$$
(\mathrm{a}, \delta)=\frac{k}{2 v}\left\{\mathrm{a}+A p-\frac{h+\Phi}{\nabla}(\mathrm{b} C r-\mathrm{c} B q)\right\}+F v-F v_{0}
$$

or putting $v=v_{0}$,

$$
(\mathrm{a}, \delta)=\frac{k}{2 v_{0}}\left\{\mathrm{a}+A p_{0}-\frac{h+\Phi_{0}}{\nabla_{0}}\left(\mathrm{~b} C r_{0}-\mathrm{c} B q_{0}\right)\right\}
$$

and therefore also

$$
\begin{aligned}
& (\mathrm{b}, \delta)=\frac{k}{2 v_{0}}\left\{\mathrm{~b}+B q_{0}-\frac{h+\Phi_{0}}{\nabla_{0}}\left(\mathrm{c} A p_{0}-\mathrm{a} C r_{0}\right)\right\} \\
& (\mathrm{c}, \delta)=\frac{k}{2 v_{0}}\left\{\mathrm{c}+C r_{0}-\frac{h+\Phi_{0}}{\nabla_{0}}\left(\mathrm{a} B q_{0}-b A p_{0}\right)\right\}
\end{aligned}
$$

C. 
Again,

$$
\begin{aligned}
(h, \delta)= & 2 p(A p, \delta)+2 q(B q, \delta)+2 r(C r, \delta) \\
(A p, \delta)= & \left(A p, \delta^{\prime}\right)+\left(A p, \delta^{\prime \prime}\right) \\
\left(A p, \delta^{\prime}\right)=\frac{k}{\kappa v}(A p, \kappa \varpi)+(A p, k) \frac{d \delta^{\prime}}{d k} & =\frac{k}{\kappa v}(A p, \kappa \varpi), \\
(A p, \kappa \varpi)=\frac{1}{2}\{ & \left(1+\lambda^{2}\right)(\kappa u+2 \lambda \varpi)-(\lambda u+\varpi) \kappa \lambda \\
& +(\lambda \mu+\nu)(\kappa v+2 \mu \varpi)-(\lambda v-w) \kappa \mu \\
& +(\nu \lambda+\mu)(\kappa w+2 \nu \varpi)-(v+\lambda w) \kappa \nu\} \\
= & \frac{1}{2} \kappa(u+\lambda \varpi)=\frac{1}{2} \kappa(a+A p) \ldots \ldots \ldots \ldots \ldots \ldots
\end{aligned}
$$

therefore

$$
\begin{aligned}
\left(A p, \delta^{\prime}\right) & =\frac{k}{2 u}(\mathrm{a}+A p) \\
\left(A p, \delta^{\prime \prime}\right) & =-k \frac{h+\Phi}{v \nabla}(A p, v)+\& \mathrm{c} . \\
& =-\frac{1}{2} k \frac{h+\Phi}{v \nabla}(\mathrm{b} C r-\mathrm{c} B q)+F v-F v_{0}, \\
(A p, \delta) & =\frac{k}{2 v}\left\{\mathrm{a}-A p-\frac{h+\Phi}{\nabla}(\mathrm{b} C r-\mathrm{c} B q)\right\}+F v-F v_{0},
\end{aligned}
$$

and similarly for $(B q, \delta),(C r, \delta)$. Substituting, and neglecting the terms which vanish for $v=v_{0}$,

$$
(h, \delta)=\frac{k}{v}\left(\Phi+h-\frac{\Phi+h}{\nabla} \nabla\right)
$$

i.e.

$$
(h, \delta)=0
$$

Lastly, to find $(\epsilon, \delta)$,

$$
(\epsilon, \delta)=\{(\epsilon, \delta)\}+(\mathrm{a}, \delta) \frac{d \epsilon}{d \mathrm{a}}+(\mathrm{b}, \delta) \frac{d \epsilon}{d \mathrm{~b}}+(\mathrm{c}, \delta) \frac{d \epsilon}{d \mathrm{c}},
$$

where, in $\{(\epsilon, \delta)\}$, the differentiations upon $\epsilon$ are supposed not to affect the constants $\mathrm{a}, \mathrm{b}, \mathrm{c}$. Neglecting the terms which vanish for $v=v_{0}$,

$$
\begin{aligned}
(\epsilon, \delta) & =\{(\epsilon, \delta)\} \\
\{(\epsilon, \delta)\} & =\left\{\left(\epsilon, \delta^{\prime}\right)\right\}+\left\{\left(\epsilon, \delta^{\prime \prime}\right)\right\} \\
\left\{\left(\epsilon, \delta^{\prime}\right)\right\} & =\left[\left\{\left(\epsilon, \delta^{\prime}\right)\right\}\right]+(\epsilon, k) \frac{d \delta^{\prime}}{d k}=\left[\left\{\left(\epsilon, \delta^{\prime}\right)\right\}\right]
\end{aligned}
$$

where, in $\left[\left\{\left(\epsilon, \delta^{\prime}\right)\right\}\right]$, the differentiations upon $\epsilon$ and $\delta$ do not affect the constants.

$$
\left\{\left(\epsilon, \delta^{\prime \prime}\right)\right\}=\left[\left\{\left(\epsilon, \delta^{\prime \prime}\right)\right\}\right]+\left(\epsilon, \text { a) } \frac{d \delta^{\prime \prime}}{d a}+\& c .\right.
$$

i.e.

$$
\left\{\left(\epsilon, \delta^{\prime \prime}\right)\right\}=\left[\left\{\left(\epsilon, \delta^{\prime \prime}\right)\right\}\right] \text { : }
$$


neglecting the terms which vanish for $v=v_{0}$,

therefore

$$
\begin{aligned}
(\epsilon, \delta) & =\left[\left\{\left(\epsilon, \delta^{\prime}\right)\right\}\right]+\left[\left\{\left(\epsilon, \delta^{\prime \prime}\right)\right\}\right] \\
& =\left[\left\{\left(\epsilon, \delta^{\prime}\right)\right\}\right] ;
\end{aligned}
$$

since

$$
\left[\left\{\left(\epsilon, \delta^{\prime \prime}\right)\right\}\right]=(v, v) \frac{d \epsilon}{d v} \frac{d \delta^{\prime \prime}}{d v}=0
$$

Hence

$$
(\epsilon, \delta)=-\frac{1}{2} \frac{k}{\kappa \nabla v}(v, \kappa \omega)
$$

$$
\begin{aligned}
& (\nu, \kappa \varpi)=\frac{1}{2}\left(\{u+(1+\kappa) \lambda \varpi\}(2 \lambda \varpi+\kappa u)-\left\{\lambda \varpi^{2}+(1+\kappa) \varpi u\right\} \kappa \lambda\right) \\
& +\{v+(1+\kappa) \mu \varpi\}(2 \mu \varpi+\kappa v)-\left\{\mu \varpi^{2}+(1+\kappa) \varpi v\right\} \kappa \mu \\
& \left.+\{w+(1+\kappa) \nu \varpi\}(2 \nu \varpi+\kappa w)-\left\{\nu \varpi^{2}+(1+\kappa) \varpi w\right\} \kappa \nu\right) \\
& =\frac{1}{2}\left\{2 \varpi^{2}+\kappa\left(u^{2}+v^{2}+w^{2}\right)+2(1+\kappa)(\kappa-1) \varpi^{2}+\kappa(1+\kappa) \varpi^{2}\right. \\
& \left.-\kappa(\kappa-1) \varpi^{2}-\kappa(\kappa+1) \varpi^{2}\right\} \\
& =\frac{1}{2} \kappa\left\{(\kappa+1) \varpi^{2}+\left(u^{2}+v^{2}+w^{2}\right)\right\}=\frac{1}{2} 4 \kappa v=2 \kappa v
\end{aligned}
$$

therefore

$$
(\epsilon, \delta)=-\frac{k}{\nabla_{0}}
$$

Hence, recapitulating,

$$
\begin{aligned}
& (\mathrm{b}, \mathrm{c})=-\mathrm{a}, \quad(\mathrm{c}, \mathrm{a})=-\mathrm{b}, \quad(\mathrm{a}, \mathrm{b})=-\mathrm{c}, \\
& (\mathrm{a}, h)=0, \quad(\mathrm{~b}, h)=0, \quad(\mathrm{c}, h)=0 \\
& (\mathrm{a}, \epsilon)=-\frac{1}{\nabla_{0}}\left(\mathrm{~b} C r_{0}-\mathrm{c} B q_{0}\right) \\
& (\mathrm{b}, \epsilon)=-\frac{1}{\nabla_{0}}\left(\mathrm{c} A p_{0}-\mathrm{a} C r_{0}\right), \\
& (\mathrm{c}, \epsilon)=-\frac{1}{\nabla_{0}}\left(\mathrm{a} B q_{0}-\mathrm{b} A p_{0}\right) \\
& (h, \epsilon)=-2 \\
& (\mathrm{a}, \delta)=\frac{k}{2 v_{0}}\left\{\mathrm{a}+A p_{0}-\frac{h+\Phi_{0}}{\nabla_{0}}\left(\mathrm{~b} C r_{0}-\mathrm{c} B q_{0}\right)\right\} \\
& (\mathrm{b}, \delta)=\frac{k}{2 v_{0}}\left\{\mathrm{~b}+B q_{0}-\frac{h+\Phi_{0}}{\nabla_{0}}\left(\mathrm{c} A p_{0}-\mathrm{a} C r_{0}\right)\right\} \\
& (\mathrm{c}, \delta)=\frac{k}{2 v_{0}}\left\{\mathrm{c}+C r_{0}-\frac{h+\Phi_{0}}{\nabla_{0}}\left(\mathrm{a} B q_{0}-\mathrm{b} A p_{0}\right)\right\} \\
& (h, \delta)=0 \\
& (\epsilon, \delta)=-\frac{k}{\nabla_{0}}
\end{aligned}
$$


and therefore

$$
\begin{gathered}
\frac{d \mathrm{a}}{d t}=-\mathrm{c} \frac{d V}{d \mathrm{~b}}+\mathrm{b} \frac{d V}{d \mathrm{c}}-\frac{1}{\nabla_{0}}\left(\mathrm{~b} C r_{0}-\mathrm{c} B q_{0}\right) \frac{d V}{d \epsilon}+\frac{k}{2 v_{0}}\left\{\mathrm{a}+A p_{0}-\frac{h+\Phi_{0}}{\nabla_{0}}\left(\mathrm{~b} C r_{0}-\mathrm{c} B q_{0}\right)\right\} \frac{d V}{d \delta} \\
\frac{d \mathrm{~b}}{d t}=-\mathrm{a} \frac{d V}{d \mathrm{c}}+\mathrm{c} \frac{d V}{d \mathrm{a}}-\frac{1}{\nabla_{0}}\left(\mathrm{c} A p_{0}-\mathrm{a} C r_{0}\right) \frac{d V}{d \epsilon}+\frac{k}{2 v_{0}}\left\{\mathrm{~b}+B q_{0}-\frac{h+\Phi_{0}}{\nabla_{0}}\left(\mathrm{c} A p_{0}-\mathrm{a} C r_{0}\right)\right\} \frac{d V}{d \delta} \\
\frac{d \mathrm{c}}{d t}=-\mathrm{b} \frac{d V}{d \mathrm{a}}+\mathrm{a} \frac{d V}{d \mathrm{~b}}-\frac{1}{\nabla_{0}}\left(\mathrm{a} B q_{0}-\mathrm{b} A p_{0}\right) \frac{d V}{d \epsilon}+\frac{k}{2 v_{0}}\left\{\mathrm{c}+C r_{0}-\frac{h+\Phi_{0}}{\nabla_{0}}\left(\mathrm{a} B q_{0}-\mathrm{b} A p_{0}\right)\right\} \frac{d V}{d \delta} \\
\frac{d h}{d t}=-2 \frac{d V}{d \epsilon}, \\
\frac{d \epsilon}{d t}=\frac{1}{\nabla_{0}}\left\{\left(\mathrm{~b} C r_{0}-\mathrm{c} B q_{0}\right) \frac{d V}{d \mathrm{a}}+\left(\mathrm{c} A p_{0}-\mathrm{a} B q_{0}\right) \frac{d V}{d \mathrm{~b}}+\left(\mathrm{a} B q_{0}-\mathrm{b} A p_{0}\right) \frac{d V}{d \mathrm{c}}\right\}+2 \frac{d V}{d h}-\frac{k}{\nabla_{0}} \frac{d V}{d \delta} \\
\frac{d \delta}{d t}=-\frac{k}{2 v_{0}}\left[\quad\left\{\mathrm{a}+A p_{0}-\frac{h+\Phi_{0}}{\nabla_{0}}\left(\mathrm{~b} C r_{0}-\mathrm{c} B q_{0}\right)\right\} \frac{d V}{d \mathrm{a}}\right. \\
+\left\{\mathrm{b}+B q_{0}-\frac{h+\Phi_{0}}{\nabla_{0}}\left(\mathrm{c} A p_{0}-\mathrm{a} C r_{0}\right)\right\} \frac{d V}{d \mathrm{~b}} \\
\left.+\left\{\mathrm{c}+C r_{0}-\frac{h+\Phi_{0}}{\nabla_{0}}\left(\mathrm{a} B q_{0}-\mathrm{b} A p_{0}\right)\right\} \frac{d V}{d \mathrm{c}}\right]+\frac{k}{\nabla_{0}} \frac{d V}{d \epsilon}
\end{gathered}
$$

to which we may join

$$
\frac{d k}{d t}=\frac{d V}{d \delta}
$$

We have thus the complete system of formulæ. 